\title{
Probiotics modify human intestinal mucosa-associated microbiota in patients with colorectal cancer
}

\author{
ZHIGUANG GAO*, BOMIN GUO*, RENYUAN GAO*, QINGCHAO ZHU, WEN WU and HUANLONG QIN
}

Department of General Surgery, Shanghai Jiao Tong University Affiliated Sixth People's Hospital, Shanghai 200233, P.R. China

Received October 13, 2014; Accepted June 23, 2015

DOI: $10.3892 / \mathrm{mmr} .2015 .4124$

\begin{abstract}
Studies using animal models have demonstrated that probiotics may have a beneficial role in the prevention of colorectal cancer (CRC); however, the underlying mechanism of the beneficial effects of interventional probiotic treatment on gut microbiota has remained elusive. In the present study, pyrosequencing of the $\mathrm{V} 3$ region of the 16S rRNA genes was conducted in order to determine the extent to which probiotics alter the microbiota. The observations of the present study indicated that the microbial structure of cancerous tissue differed significantly from that of healthy individuals and that the CRC microbiota exhibited lower diversity. It was indicated that interventional treatment with probiotics increased the density and diversity of mucosal microbes, and altered the mucosa-associated microbiota. Pyrosequencing demonstrated that probiotics significantly reduced (5-fold) the abundance of a bacterial taxon assigned to the genus Fusobacterium, which had been previously suggested to be a contributing factor to increase tumorigenesis. Accordingly, interventional probiotic therapy is suggested to be able to improve the composition of the mucosal microbial flora and significantly reduce the abundance of mucosa-associated pathogens in patients with CRC.
\end{abstract}

\section{Introduction}

Colorectal cancer (CRC) is one of the most common malignancies and the incidence has increased significantly in recent years (1), particularly in developing countries such as China, where the annual increase of $4.71 \%$ exceeds the global increase rate of $2 \%$ (2). To date, the etiology and pathogenesis of CRC have remained to be fully elucidated. Previous studies have suggested that genetic factors are involved in $5-15 \%$ of all

Correspondence to: Dr Huanlong Qin, Department of General Surgery, Shanghai Jiao Tong University Affiliated Sixth People's Hospital, 600 Yishan Road, Shanghai 200233, P.R. China

E-mail: huanlongqin_123@163.com

*Contributed equally

Key words: probiotics, mucosa-associated microbiota, colorectal cancer, Fusobacterium, dysbiosis cases of CRC (3). The majority of the remaining cases, which are classified as sporadic CRC, are affected by environmental factors (4), including age, gender, diet, obesity and levels of physical inactivity. One of the common characteristics of these potential risk factors is that they can cause alterations in the structure of the intestinal flora (5-8). These alterations (also called dysbiosis) were hypothesized to be closely correlated with the pathogenesis of CRC (9-11).

The human intestine harbors $\sim 10^{14}$ microbes, whose genetic content is 100 times higher than that of the human genome (12). The intestine and its microbe population are collectively referred to as a super-organism, which constitutes a large and complex ecosystem, and is directly involved in functions including digestion and nutrient absorption, energy supply, fat metabolism, immune regulation and disease resistance $(13,14)$. The microflora in the human gut contains beneficial commensal bacteria and certain potential pathogenic bacteria. These two types of bacteria interact with their human host, leading to physiological inflammation, which can prevent diseases including inflammatory bowel disease (IBD) and CRC. However, when these potentially pathogenic bacteria increase in number or their virulence is enhanced, this physiological inflammation is likely to become a pathological inflammation and possibly cancer-causing $(15,16)$. Accordingly, the developments of methods to increase the number of beneficial bacteria or suppress harmful bacteria would be advantageous (17).

The term probiotics refers to preparations containing a class of active microorganisms in the human intestine that can bring health benefits to the host, such as improvement of the host micro-ecological balance $(18,19)$. The majority of the probiotics available at present include lactic acid bacteria (20), in addition to the genera Lactobacillus, Bifidobacterium and Enterococcus (21). Studies in animal models and humans have demonstrated that the consumption of probiotics is effective in various medical conditions, can delay senility (22), and improve acute infectious diarrhea $(23,24)$, antibiotic-associated diarrhea $(21,25)$, constipation (26-28), ulcerative colitis $(29,30)$ and Crohn's disease $(31,32)$. However, the majority of these previous studies focused on the interaction between probiotics and the mammalian host. It has remained elusive whether probiotics serve a beneficial role by adjusting intestinal microbes or inhibiting certain detrimental pathogens. In the light of these previous studies, it was suggested that it may be feasible to reduce the incidence of CRC by the application 
of probiotics and optimization of the gut microflora (17). The aim of the present study was to assess whether perioperative oral probiotics were able to alter the microbial composition and improve gut microecology in patients with CRC.

\section{Materials and methods}

Patients. A total of 60 patients with CRC who were due to undergo radical colorectomy at Shanghai Jiao Tong University Affiliated Shanghai Sixth People's Hospital (Shanghai, China), were selected between September 2011 and December 2013. The inclusion and exclusion criteria are listed in (Table I). A total of 60 patients were assessed for eligibility; however, only 38 were finally enrolled due to the fact that 22 failed to meet the inclusion criteria or refused to participate in the study. A total of 22 patients completed the trial (Fig. 1). All eligible patients $(n=22)$ were randomized and divided into two treatment groups: The CGT group $(\mathrm{n}=11)$ received perioperative placebos and the PGT group $(\mathrm{n}=11)$ received probiotics. There were no significant differences in gender, age, body mass index (BMI), cancer stage and time between onset of symptoms and hospital admission between the two groups. No significant differences in the levels of albumin, hemoglobin or creatinine were observed between the two groups (Table II).

In order to analyze further differences in the microbiota between patients with CRC and the healthy population, an additional 11 healthy volunteers (HGT group) were enrolled, who were part of a larger population-based case-control study at the Shanghai Jiao Tong University (Table II). The inclusion and exclusion criteria for the controls are listed in Table I.

Study design, probiotics treatment and patient care. A single-center prospective randomized control study design was used. Equal randomization was accomplished using a computer-generated random allocation schedule. The probiotic and placebo were sealed with aluminum foil and appeared identical in the two groups, with an identical smell and taste. Only one nurse not directly involved in the trial was able to solve the treatment codes in the event of an emergency. An acid-resistant coating was used to prepare the capsules containing the probiotics and placebo. Patients in the PGT group received an encapsulated probiotics preparation (Shanghai Xinyi Pharmaceutical Co., Ltd., Shanghai, China) containing live combined Bifidobacterium longum, Lactobacillus acidophilus and Enterococcus faecalis (1:1:1) with no less than $1.0 \times 10^{7} \mathrm{CFU} / \mathrm{g}$ viable cells, three times/day, with a total daily dose of $6.0 \times 10^{7} \mathrm{CFU}$ for five days. Patients in the CGT group received encapsulated maltodextrin (Haiyan Liuhe Pharmaceutical Industry Co., Ltd., Jiangsu, China) three times a day. During the study period, no parenteral or enteral nutritional supplementation was administered. All patients received a regular diet preoperatively and conventional bowel preparation was performed on pre-operative day 1 , including the administration of a low-residue diet and mechanical bowel preparation. All patients were given Soffodex (Jiangxi Hygecon Pharmaceutical Co., Shangrao, China), containing $2.4 \mathrm{~g}$ monobasic sodium phosphate and $0.9 \mathrm{~g}$ dibasic sodium phosphate. Parenteral hydration was administered on the morning of the surgery supplied via a central venous catheter. A $12 \mathrm{~F}$ catheter was placed through a jejunal limb during surgery for gastric aspiration to reduce colon anastomotic fluxion. For prophylaxis, $500 \mathrm{mg}$ metronidazole (Sine Wanxiang Pharmaceutical Co., Shanghai, China) and $1 \mathrm{~g}$ ceftriaxone (Shanghai Roche Pharmaceutical Co., Shanghai, China) were administered $1 \mathrm{~h}$ prior to induction and continued for $48 \mathrm{~h}$ following the operation. All subjects were interviewed by the study nurse, and reactions to the product, medications taken and adverse events that occurred during the five-day period were recorded.

Healthy subjects underwent a standard bowel cleansing preparation with oral polyethylene glycol electrolyte (139.12 g/2,000 ml; Jiangxi Hygecon Pharmaceutical Co.) the evening prior to colonoscopy. Endoscopically, the colonic mucosa appeared normal in all subjects. Colonic mucosal tissue biopsies $(\sim 1 \times 2 \mathrm{~mm}$ each) were collected from the ascending colon $(n=3)$, descending colon $(n=1)$, sigmoid colon $(n=3)$ and rectum $(n=4)$ during colonoscopy.

Ethics statement. The present study was approved by the Ethics Committee of the Sixth People's Hospital Affiliated to Shanghai Jiaotong University on Human Subjects in Medical Research [protocol number 2011 (L)-11] and all individuals provided written informed consent prior to participating in the study.

Sample collection and DNA extraction. Colorectal mucosa tissue samples were collected from the tumor sites during surgery. Normal colorectal mucosa tissue samples were collected from the cecum, ascending colon, transverse colon, descending colon, sigmoid colon, and rectum during colonoscopy. Fisher's exact test was used for comparison of different anatomic sites. All samples from the study participants, including healthy individuals, were placed in liquid nitrogen immediately and transported to the laboratory within 30 min of collection. DNA was extracted from all intestinal tissue samples using MoBio Powersoil DNA extraction kits (MoBio Laboratories, Inc., Carlsbad, CA, USA), according to the manufacturer's instructions. Intestinal tissue samples were completely lysed by overnight incubation at $56^{\circ} \mathrm{C}$ in buffer ATL (Qiagen, Düsseldorf, Germany) and proteinase K (Shanghai SAIBAISHENG Gene Technology Co., Ltd., Shanghai, China). The standard tissue protocol was modified to extend the $70^{\circ} \mathrm{C}$ incubation step from 10 to $30 \mathrm{~min}$.

DNA was eluted from a column in a final volume of $200 \mu \mathrm{l}$ elution buffer and stored at $-20^{\circ} \mathrm{C}$ prior to the amplification steps.

Bacterial $16 S \mathrm{r}$ RNA amplification and pyrosequencing. The V3 region of the bacterial 16S rRNA gene was amplified from extracted DNA using primers (Sangon Biotech Co., Ltd., Shanghai, China) 27F (5'-AGAGTTTGATCCTGGCTC AG-3') and 533R (5'-TTACCGCGGCTGCTGGCAC-3') (33), with a sample barcode sequence and the FLX Titanium adapters (Roche Diagnostics, Basel, Switzerland) were used to amplify the $\mathrm{V} 3$ region of each tissue sample by polymerase chain reaction (PCR). The PCR mixture was composed as follows: $10 \mathrm{ng}$ template, $0.4 \mathrm{ml}$ FastPfu Polymerase (Beijing TransGen Biotech Co., Ltd., Beijing, China), 4 ml 56 FastPfu buffer (Transgene, Illkirch-Graffenstaden, France), $2 \mathrm{ml}$ deoxynucleotide diphosphate $(2.5 \mathrm{mM}$ each; Takara Bio, Inc., Otsu, Japan), $0.4 \mathrm{ml}$ forward primers $(5 \mathrm{mM})$ and $0.4 \mathrm{ml}$ reverse primers $(5 \mathrm{mM})$. The reaction was performed 
Table I. Inclusion and exclusion criteria for enrolment of individuals in the present study.

Colorectal cancer patients

Healthy individuals

\section{Inclusion criteria}

Age 40-75 years

Diagnosis confirmed by biopsy and histological analysis

Previous radical resection and no distant

metastasis (including liver)

Exclusion criteria

Age $>75$ years

Pregnancy

Known lactose intolerance

Clinically significant immunodeficiency

Usage of antibiotics and additional gastrointestinal

disorders (e.g. Crohn's disease or ulcerative colitis)

Received antibiotics for the past 3 months prior to surgery

Evidence of infection

Probiotics or prebiotics and excessive fiber intake

within 2 weeks

Patient subjected to emergency operation

Bowel preparation for colonoscopy within 6 days

prior to surgery

Patient subjected to proctectomy with low rectal

anastomosis or surgery for polypoid lesion

Laparoscopic surgery

Patients received preoperative chemotherapy

or radiation therapy
Inclusion criteria

Age 40-75 years

BMI 18.5-30 kg/m²

Exclusion criteria

$\mathrm{BMI}>30 \mathrm{~kg} / \mathrm{m}^{2}$

Pregnancy

Known lactose intolerance

Clinically significant immunodeficiency

Usage of antibiotics and additional gastrointestinal disorders

(e.g. Crohn's disease or ulcerative colitis)

Received antibiotics for the past 3 months prior to surgery

Evidence of infection

Probiotics or prebiotics and excessive fiber intake

within 2 weeks

Patient subjected to emergency operation

Bowel preparation for colonoscopy within 6 days prior to surgery

Patient subjected to proctectomy with low rectal anastomosis or surgery for polypoid lesion

Laparoscopic surgery

History or presence of other tumors

BMI, body mass index.

in an ABI GeneAmp H 9700 Cycler (Applied Biosystems Life Technologies, Foster City, CA, USA) and the cycling parameters were as follows: $5 \mathrm{~min}$ denaturation at $95^{\circ} \mathrm{C}$ followed by 25 cycles of $30 \mathrm{sec}$ at $95^{\circ} \mathrm{C}$ (denaturation), $30 \mathrm{sec}$ for annealing at $55^{\circ} \mathrm{C}$ and $30 \mathrm{sec}$ at $72^{\circ} \mathrm{C}$ (elongation), with a final extension at $72^{\circ} \mathrm{C}$ for $5 \mathrm{~min}$. Triplicate PCR reactions were performed on each sample. Amplified products from stool samples were verified by gel electrophoresis using $5 \mathrm{ml}$ PCR reaction mixture in a $2.0 \%$ agarose gel. The PCR products were purified using the AxyPrepDNA Gel extraction kit (Corning Life Sciences, Corning, NY, USA) and quantified on a QuantiFluor ${ }^{\mathrm{TM}}$-ST Fluorometer (Promega Corporation, Madison, WI, USA). The products from different samples were mixed at equal ratios for pyrosequencing using the Roche GS FLX 454 Sequencer (Roche Diagnostics) according to the manufacturer's instructions. All pyrosequencing reads were then removed of their primers, barcodes and adaptor sequences, and were further screened and filtered according to the standards for quality control as follows: The elimination of sequences that did not perfectly match the proximal PCR primer (over two mismatches to the primers), those with short sequencing length $(<200$ nucleotides) sequences that contained mononucleotide repeats of six nucleotides, sequences with ambiguous characters, or sequences with a read quality score $<25$. Finally, a total of 416,599 high-quality sequences from 22 samples were produced, which accounted for $72.9 \%$ of valid sequences according to barcode- and primer-sequence filtering.

Bioinformatics analysis of sequencing data. In order to gain high-quality and more precise bioinformation, effective sequences that contained certain point mutations and macromolecular homopolymers were narrowed down using QIIME, version 1.17 (http://qiime.org/) based on the following methods and parameters: Number of mismatching of primers, $<2$; maximum number of 3' primers, 3; no ambiguous bases or $>6$ homologous mono-bases; and sequence length, $<200$ base pairs (34). The optimized sequences were then clustered into operational taxonomic units (OTUs) using Usearch, version 7.1 (http://drive5.com/uparse/)withacriterion of minimumsimilarity of $97 \%$. Chimera sequences arising from the PCR amplification were detected and excluded from the OTUs using UCHIME version 4.2.40 (http://drive5.com/usearch/manual/uchime_algo. html) (35). Using the usearch_global command, representative OTUs were aligned to the optimized sequences and the abundance of OTUs per sample was obtained for performing the following further analysis.

Applying Bayesian algorithms of ribosomal database project (RDP) classifier (http://rdp.cme.msu.edu/), representative OTUs were identifed in the following databases: 16S bacteria and archaeal ribosomes [SILVA (36); release 115; http://www. arb-silva.de]; RDP (21) (release 11.1; http://rdp.cme.msu.edu/); 
Table II. Statistical data of subjects of the present study.

\begin{tabular}{lcccr}
\hline Parameter & HGT & CGT & PGT & P-value \\
\hline Sample type & Tissue & Tissue & Tissue & \\
Number of individuals (n) & 11 & 11 & 11 & $>0.05$ \\
Male/female & $6 / 5$ & $6 / 5$ & $6 / 5$ & $>0.05$ \\
Age (years) & $71 \pm 5.4$ & $68 \pm 7.3$ & $65 \pm 9.6$ & $>0.05$ \\
BMI (kg/m ${ }^{2}$ ) & $22.2 \pm 2.2$ & $24.5 \pm 4.3$ & $24.9 \pm 4.2$ & $>0.05$ \\
Stage (A/B/C) & - & $4 / 4 / 3$ & $3 / 5 / 3$ & $>0.05$ \\
Location & 3 & & & $>0.05$ \\
Ascending colon (n) & 1 & 1 & 2 & $>0.05$ \\
Descending colon (n) & 3 & 3 & 1 & $>0.05$ \\
Sigmoid colon (n) & 4 & 5 & 6 & $>0.05$ \\
Rectum (n) & $42.2 \pm 2.6$ & $36.5 \pm 3.4$ & $40.1 \pm 3.3$ & $>0.05$ \\
Pre-operative albumin (g/dl) & $126 \pm 12.4$ & $123.2 \pm 19.6$ & $125.3 \pm 17.7$ & $>0.05$ \\
Pre-operative Hb (g/l) & $1.1 \pm 0.2$ & $1.2 \pm 0.13$ & $1.1 \pm 0.16$ & $>0.05$ \\
Creatinine (mg/dl) & & & 2 & \\
\hline
\end{tabular}

Values are expressed as the expressed as the mean \pm standard deviation. BMI, body mass index; CRC, colorectal cancer; HGT, healthy volunteer group; CGT, perioperative placebo group; PGT, probiotics group; Hb, hemoglobin.

Greengene (37) (release 13.5; http://greengenes.secondgenome. com/); ITS fungus [Unite(33); release 5.0; http://unite.ut.ee/index. php]; and functional genes (FGR; release 7.3; http://fungene. cme.msu.edu/). Subsequently, analysis with Good's coverage estimator, diversity estimators (Shannon and Simpson), richness estimators [Chao and abundance-based coverage estimators (ACE)] were performed and the rarefaction curve was generated using the Mothur software package (http://www.mothur. org/wiki/Main_Page) at an 80\% confidence level. In addition, the Bray-Curtis dissimilarities were used to construct a cluster dendrogram. A metagenomic biomarker discovery approach was employed with linear discriminant analysis (LDA) coupled with effect size measurement (LEfSe), which was used to perform a non-parametric Wilcoxon sum-rank test followed by LDA analysis using online software (http://huttenhower.sph. harvard.edu/galaxy/) to assess the effect size of each differentially abundant taxon.

Statistical analysis. Results were analysed using SPSS, version 18.0 for Windows (SPSS, Inc., Chicago, IL, USA). Quantitative values are expressed as the mean \pm standard deviation. Comparison of categorical data between groups was performed using the Pearson $\chi^{2}$ test or, where indicated, Fisher's exact test. Analysis of variance or the Kruskal-Wallis test was used for continuous variables, as appropriate. $\mathrm{P}<0.05$ was considered to indicate a statistically significant difference.

Sequence data. The $16 \mathrm{~S}$ sequence data generated in the present study were submitted to the GenBank Sequence Read Archive with the accession number SRP043381.

\section{Results}

Probiotics simultaneously increase richness and diversity of mucosal microbes. A total of 416,599 high-quality, classifiable reads were obtained, with an average of $12,624 \pm 2,423(n=33)$ reads/sample. UPARSE, version 7.1 (http://drive5.com/uparse/) was used to cluster the above reads. At a 3\% dissimilarity level, 45,453 OTUs in all samples and an average of 1,377 OTUs $(n=33)$ were identified per sample.

Chao and ACE indices. The PGT group exhibited significantly higher Chao $(\mathrm{P}=0.013)$ and $\mathrm{ACE}(\mathrm{P}=0.01)$ indices in colorectal mucosa compared with those in the HGT group. There were no significant differences between the HGT and CGT groups or CGT and PGT groups (Fig. 2).

Shannon and Simpson indices. Compared with the HGT group, the Shannon $(\mathrm{P}=0.028)$ and Simpson $(\mathrm{P}=0.046)$ indices were significantly reduced in the CGT group. This demonstrated that the mucosal microflora diversity was reduced in patients with $\mathrm{CRC}$, while the diversity of the intestinal flora increased following oral administration of probiotics.

Probiotics modulate gut microflora structure. The gut microflora structure was compared at various bacterial levels. At the phylum level, there were no significant differences in classification among the three groups (27 in the CGT group, 29 in the PGT group and 25 in the HGT group). A microflora whose abundance was $>0.1 \%$ contributed to $99.8 \%$ of the total category, including Firmicutes, Bacteroidetes, Proteobacteria, Actinobacteria and Fusobacteria. At the genus level, 188 were present in the CGT group, 198 in the PGT group and 201 in the HGT group. Bacteria whose abundance was $>0.01 \%$ contributed to $96.86 \%$ of the total category in the HGT group, compared with $99.76 \%$ in the CGT group and $99.13 \%$ in the PGT group. A cladogram representation of the structure of the mucosal microbiota (Fig. 3) and a bar 
Table III. Comparison of gut microflora structure at phylum and genus levels.

\begin{tabular}{|c|c|c|c|c|}
\hline \multirow[b]{2}{*}{ Phylum/genus/species } & \multicolumn{3}{|c|}{ Relative abundance $(\%)$} & \multirow[b]{2}{*}{ P-value } \\
\hline & HGT & CGT & PGT & \\
\hline Firmicutes & $40.21^{\mathrm{a}}$ & 60.97 & $66.44^{\mathrm{a}}$ & 0.019 \\
\hline Bacillus & $35.81^{\mathrm{a}}$ & 53.83 & $63.51^{\mathrm{a}}$ & 0.017 \\
\hline Brochothrix & 3.05 & 0.12 & 0.19 & $>0.001$ \\
\hline Carnobacterium & $0.47^{\mathrm{a}}$ & 0.70 & $0.88^{\mathrm{a}}$ & 0.018 \\
\hline Enterococcus & $0.03^{\mathrm{b}}$ & $0.33^{\mathrm{a}}$ & $2.83^{\mathrm{a}, \mathrm{b}}$ & 0.033 \\
\hline Coprococcus & 0.14 & $0.61^{\mathrm{a}}$ & $0.01^{\mathrm{a}}$ & 0.020 \\
\hline Peptostreptococcus & $0.00^{\mathrm{a}}$ & $0.23^{\mathrm{a}}$ & 0.09 & 0.036 \\
\hline Bacteroidetes & 11.06 & 10.12 & 8.49 & $>0.050$ \\
\hline Flavobacteria & $8.32^{\mathrm{a}}$ & $1.83^{\mathrm{a}}$ & $2.18^{\mathrm{a}}$ & $>0.001$ \\
\hline Epilithonimonas & $0.14^{\mathrm{a}, \mathrm{b}}$ & $0.03^{\mathrm{a}}$ & $0.03^{\mathrm{b}}$ & 0.04 \\
\hline Flavobacterium & $4.67^{\mathrm{a}, \mathrm{b}}$ & $1.03^{\mathrm{a}}$ & $1.33^{\mathrm{b}}$ & $>0.001$ \\
\hline Sphingobacteria & $0.50^{\mathrm{a}}$ & $0.08^{\mathrm{a}}$ & $0.20^{\mathrm{a}}$ & 0.001 \\
\hline Pedobacter & $0.10^{\mathrm{a}}$ & $0.01^{\mathrm{a}}$ & $0.01^{\mathrm{a}}$ & 0.001 \\
\hline Sphingobacterium & $0.36^{\mathrm{a}}$ & $0.04^{\mathrm{a}}$ & $0.05^{\mathrm{a}}$ & $>0.001$ \\
\hline Proteobacteria & $46.05^{\mathrm{b}}$ & $11.55^{\mathrm{a}}$ & $19.65^{\mathrm{a}, \mathrm{b}}$ & $>0.001$ \\
\hline Alphaproteobacteria & $3.94^{\mathrm{b}}$ & $1.26^{\mathrm{a}, \mathrm{b}}$ & $3.74^{\mathrm{a}}$ & 0.032 \\
\hline Caulobacter & $1.36^{\mathrm{a}}$ & $0.02^{\mathrm{a}}$ & $0.03^{\mathrm{a}}$ & $>0.001$ \\
\hline Brevundimonas & $0.68^{\mathrm{a}}$ & $0.04^{\mathrm{a}}$ & $0.04^{\mathrm{a}}$ & $>0.001$ \\
\hline Rhizobium & $0.19^{\mathrm{a}}$ & $0.98^{b}$ & $3.29^{\mathrm{a}, \mathrm{b}}$ & 0.010 \\
\hline Sphingomonas & $0.98^{\mathrm{a}}$ & $0.06^{\mathrm{a}}$ & $0.04^{\mathrm{a}}$ & $>0.001$ \\
\hline Betaproteobacteria & $2.05^{\mathrm{a}}$ & 1.56 & $0.93^{\mathrm{a}}$ & 0.031 \\
\hline Acidovorax & $0.33^{\mathrm{a}}$ & $0.02^{\mathrm{a}}$ & $0.03^{\mathrm{a}}$ & 0.001 \\
\hline Comamonas & 0.39 & $1.04^{\mathrm{a}}$ & $0.08^{\mathrm{a}}$ & 0.007 \\
\hline Janthinobacterium & $0.54^{\mathrm{a}}$ & $0.04^{\mathrm{a}}$ & $0.05^{\mathrm{a}}$ & $>0.001$ \\
\hline Gammaproteobacteria & $39.56^{\mathrm{a}}$ & $6.66^{\mathrm{a}}$ & $14.46^{\mathrm{a}}$ & 0.001 \\
\hline Buttiauxella & $0.37^{\mathrm{a}}$ & $0.03^{\mathrm{a}}$ & $0.05^{\mathrm{a}}$ & $>0.001$ \\
\hline Escherichia-Shigella & $0.22^{\mathrm{a}}$ & 2.92 & $6.39^{\mathrm{a}}$ & 0.004 \\
\hline Rahnella & $0.66^{\mathrm{a}}$ & $0.12^{\mathrm{a}}$ & $0.24^{\mathrm{a}}$ & $>0.001$ \\
\hline Acinetobacter & $4.84^{\mathrm{a}}$ & $0.63^{\mathrm{a}}$ & $1.09^{\mathrm{a}}$ & $>0.001$ \\
\hline Enhydrobacter & $0.71^{\mathrm{a}}$ & $0.11^{\mathrm{a}}$ & $0.23^{\mathrm{a}}$ & $>0.001$ \\
\hline Psychrobacter & $0.56^{\mathrm{a}}$ & $0.05^{\mathrm{a}}$ & $0.07^{\mathrm{a}}$ & $>0.001$ \\
\hline Pseudomonas & $30.25^{\mathrm{a}}$ & $1.79^{\mathrm{a}}$ & $3.99^{\mathrm{a}}$ & $>0.001$ \\
\hline Nevskia & $0.04^{\mathrm{a}}$ & $0.03^{\mathrm{a}}$ & $0.18^{\mathrm{a}}$ & $>0.001$ \\
\hline Stenotrophomonas & $0.13^{b}$ & $0.04^{\mathrm{a}, \mathrm{b}}$ & $0.13^{\mathrm{a}}$ & 0.013 \\
\hline Fusobacteria & $0.02^{\mathrm{a}}$ & $14.75^{\mathrm{a}}$ & $1.94^{\mathrm{a}}$ & 0.030 \\
\hline Fusobacterium & $0.01^{\mathrm{a}}$ & $10.08^{\mathrm{a}}$ & 1.91 & 0.032 \\
\hline Actinobacteria & 1.91 & 1.46 & 2.58 & $>0.050$ \\
\hline Rhodococcus & $0.09^{\mathrm{a}}$ & 0.93 & $1.70^{\mathrm{a}}$ & 0.018 \\
\hline Nesterenkonia & $0.49^{\mathrm{a}}$ & $0.04^{\mathrm{a}}$ & $0.05^{\mathrm{a}}$ & 0.040 \\
\hline Propionibacterium & $0.16^{\mathrm{a}, \mathrm{b}}$ & $0.02^{\mathrm{a}}$ & $0.07^{\mathrm{b}}$ & 0.003 \\
\hline
\end{tabular}

${ }^{\mathrm{a}, \mathrm{b}} \mathrm{P}<0.05$. HGT, healthy volunteer group; CGT, perioperative placebo group; PGT, probiotics group.

graph showing the predominant bacteria (Fig. 4) were generated by LEfSe. The greatest differences in taxa between the three communities are displayed. Pyrosequencing data demonstrated that probiotics-treated patients exhibited a significant reduction in Peptostreptococcus, Comamonas,
Fusobacterium and expansion of Enterococcus and Proteobacteria in the mucosa-adherent microbiota.

Reduction of a fusobacterium bacterial group is associated with probiotics administration. To determine whether 


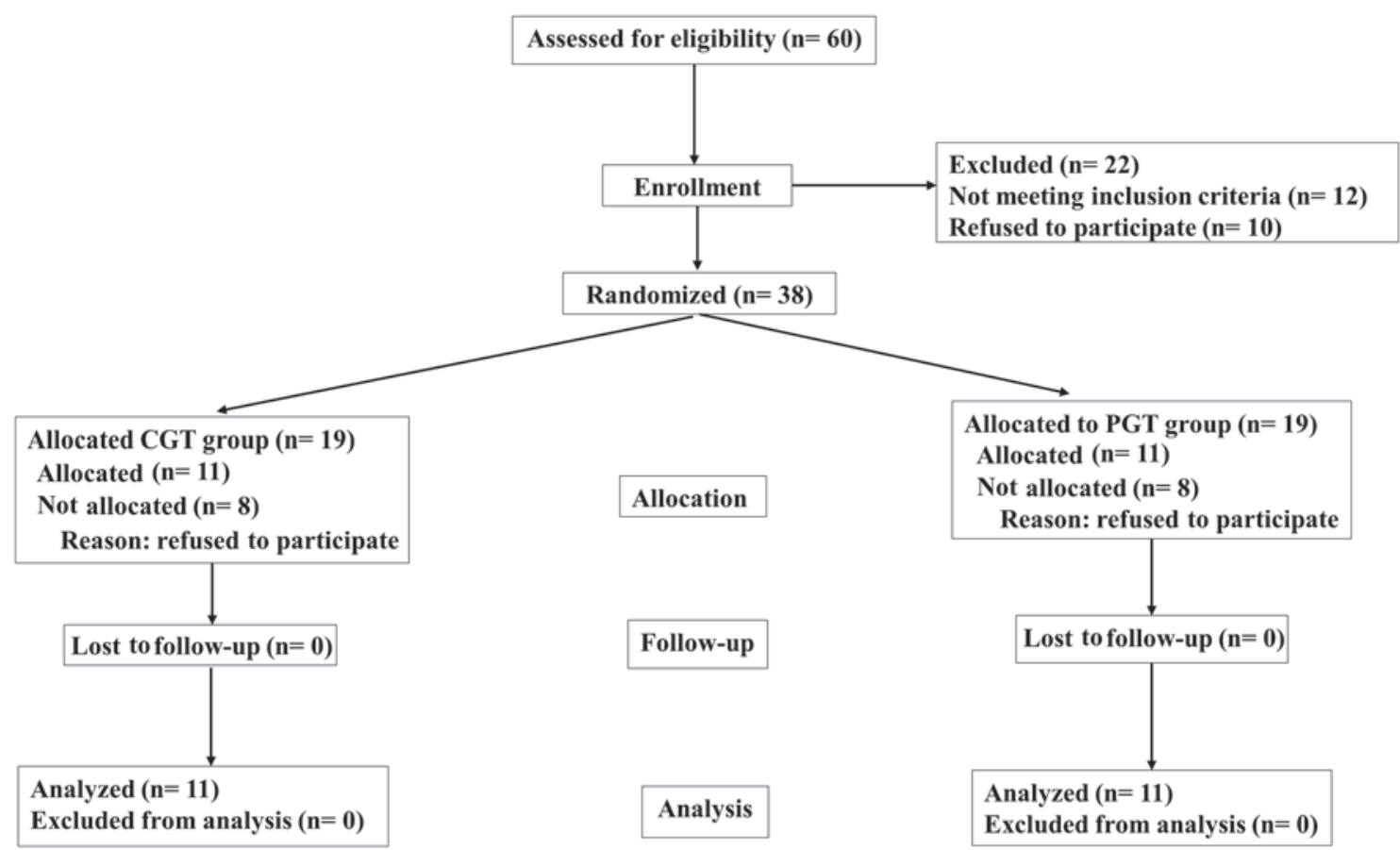

Figure 1. Flowchart of the randomization procedure used to enroll patients in the present study. CGT, perioperative placebo group; PGT, probiotics group.
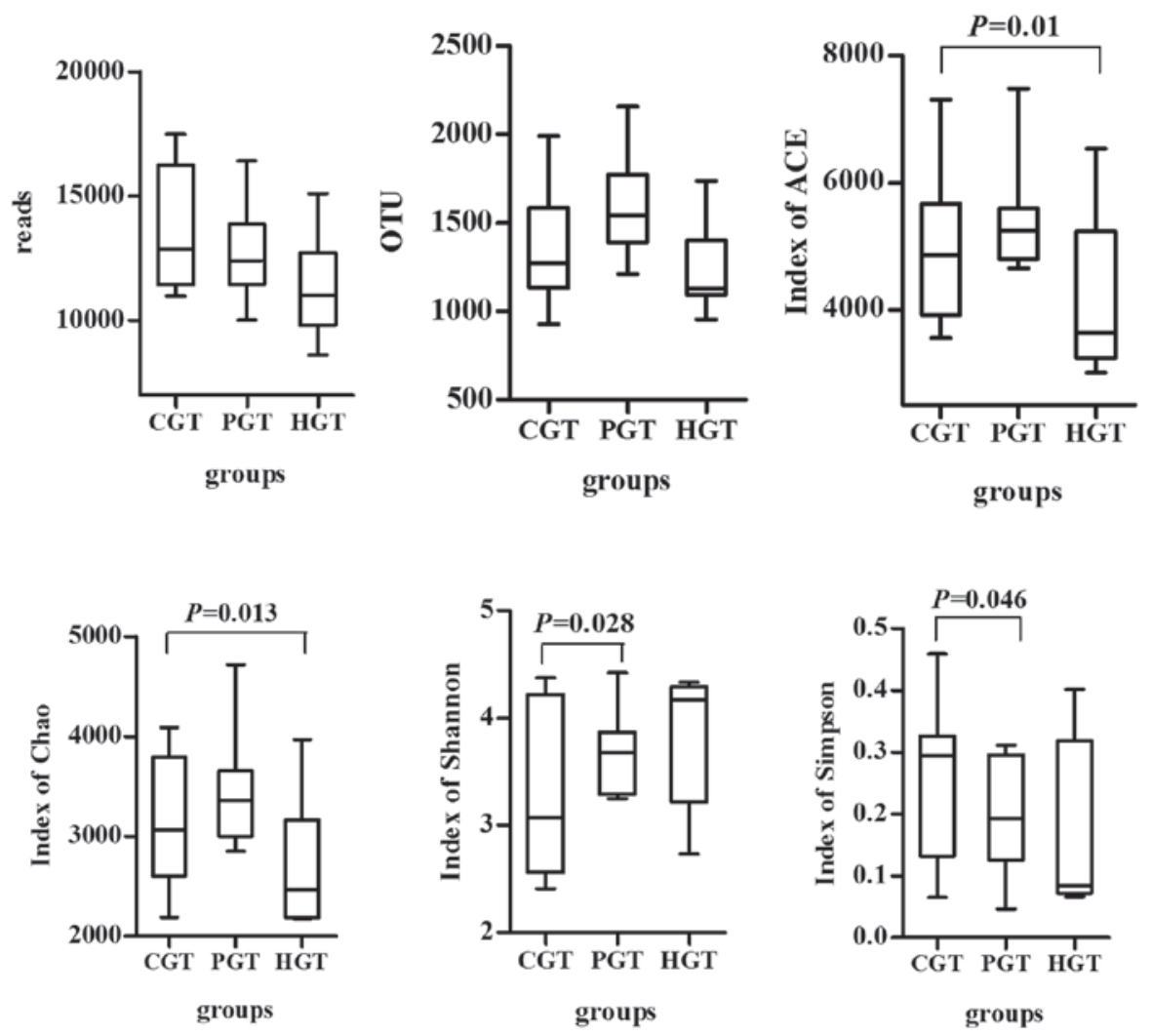

Figure 2. $\alpha$-diversity comparison using phylotype relative abundance measurements between the CGT, PGT and HGT groups. CGT, perioperative placebo group; PGT, probiotics group; HGT, healthy volunteer group; ACE, abundance-based coverage estimators; OTU, operational taxonomic units.

microbial alterations in the mucosa-associated microbiota mediate the effects of probiotics in CRC, a taxonomy-based comparison was performed to determine the differences between the microbiota of CRC and healthy individuals (Table III). Fusobacterium, which constitutes less than $0.1 \%$ of total bacteria in healthy mucosal tissue, was the most prevalent in the mucosa of patients with CRC (10.08 vs. $0.01 \%$; $\mathrm{P}=0.03$ ). In addition, probiotics treatment was observed to be associated with a significant ( $\sim$-fold) reduction in the relative abundance of Fusobacterium (1.91\%; $\mathrm{P}=0.03)$. Accordingly, 


\section{Cladogram}

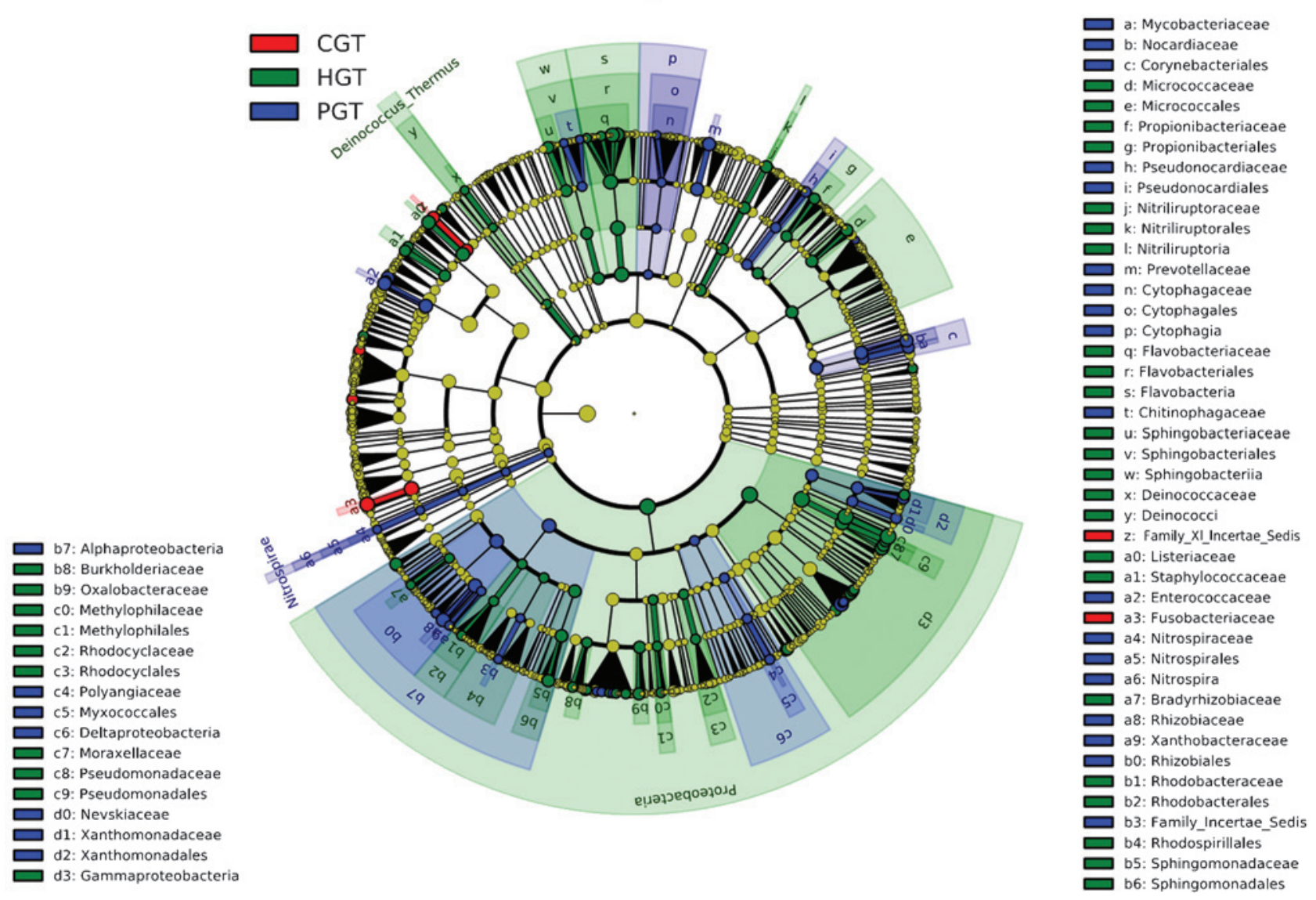

Figure 3. 16S rRNA sequencing analysis of the gut microbiota in the CGT, PGT and HGT groups. A cladogram representation of data among the three groups. The brightness of each dot is proportional to its effect size. Differences are represented by the color of the most abundant class (red indicating the CGT group, green indicating the HGT group and blue indicating the PGT group). The diameter of each circle is proportional to the abundance of the taxon. HGT, healthy volunteer group; CGT, perioperative placebo group; PGT, probiotics group.

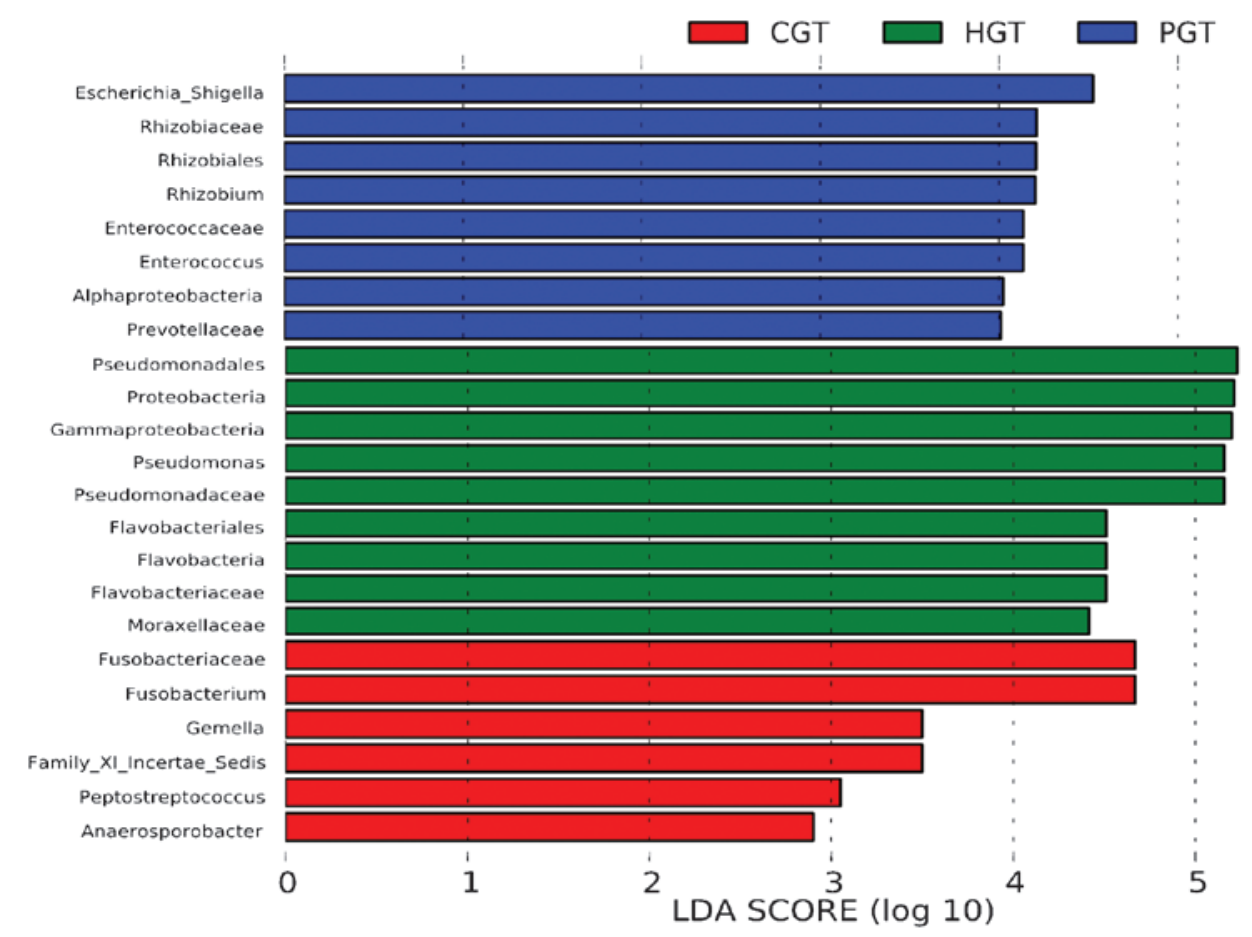

Figure 4. Different structures of the gut microbiota in the CGT, PGT and HGT groups. Histogram of the linear discriminant analysis scores for differentially abundant genera. The cladogram was calculated by LDA and displayed according to effect size. CGT, perioperative placebo group; PGT, probiotics group; linear discriminant analysis; HGT, healthy volunteer group; LDA, linear discriminant analysis. 
it was concluded that a reduction of the mucosa-associated Fusobacterium group may be a potential mediator of the effects of probiotics on CRC.

\section{Discussion}

Numerous studies have demonstrated that consumption of certain probiotics or prebiotics, which have been applied to various medical conditions, is able to favorably alter the pre-disposing factors of CRC, and is a promising approach for prevention of CRC (38). In vitro studies have demonstrated that lactic acid bacteria significantly reduce the growth and viability of the human colon cancer cell line HT-29 (39). An in vivo study in laboratory animals have additionally demonstrated that L. acidophilus and B. longum are able to reduce DNA damage and protect against 1,2-dimethylhydrazine-induced genotoxicity (40). However, unlike in vitro behavior, commensal bacteria that inhabit the human intestine are characteristic of relative stability and colonization resistance. This symbiotic flora can exclude foreign microbes from habitat. Accordingly, probiotics may serve a beneficial role by adjusting the microfloral structure rather than directly interacting with intestinal epithelial cells. To date, there have been few human studies supporting the benefits of oral administration of probiotics in high-risk populations with CRC (41).

It is of importance for a healthy intestinal microecosystem to maintain bacterial diversity (42). In the present study, the results demonstrated an enhanced abundance of the gut microflora following intake of prebiotics. A lower diversity was present in the gut microflora in patients with CRC, which was associated with the increased abundance of certain pathogens. The results were consistent with those of previous studies (38). Probiotic treatment of patients with CRC can improve the abundance of gut microflora by enhancing the diversity of the microbiota to approach a normal level (43). A possible explanation for this association is that probiotics (B. longum, L. acidophilus and E. faecalis) are able to quantitatively and/or qualitatively alter the intestinal microflora and normalize dysbiosis.

Linear discriminant analysis has indicated that certain potential pathogens, including Fusobacterium (44) and Peptostreptococcus (45) sequences, are significantly enriched in the CRC mucosal microflora. However, other bacteria, mostly belonging to the phylum of Proteobacteria, are reduced in CRC (46). It is reported that Fusobacterium may be associated with IBD, which includes ulcerative colitis and Crohn's disease (47), while IBD is one of the three highest risk factors of CRC. It is suggested that these potential pathogens or beneficial bacteria can be seen as the 'core microflora' and are directly involved in the development of CRC (48). The analysis of the present study indicated that administration of probiotics is able to effectively reduce pathogenic Fusobacterium and Peptostreptococcus populations in patients with CRC. This result agrees with a previous study, which used the Simulator of the Human Intestinal Microbial Ecosystem model, in which L. acidophilus or Lactobacillus case $i$ were demonstrated to increase lactic acid bacteria, with a concomitant reduction in fecal coliforms and clostridia (49).
Taken together, the observations of the present study demonstrated that probiotic supplements are able to effectively alter the composition, richness and diversity of the gut microflora, inhibit certain potential pathogens including Fusobacterium and Peptostreptococcus, and increase the number of certain beneficial microorganisms. Although short-term administration of probiotics cannot achieve a significant clinical effect, the results of the present study provided a basis for microbiota-associated prevention, diagnosis, prognosis and the development of treatment strategies for CRC.

\section{Acknowledgements}

The present study was supported by grants from the National Natural Science Foundation of China (grant no. 81230057) and Shen Kang Hospital Development Center of Shanghai (grant no. SHDC12012106).

\section{References}

1. Siegel R, Naishadham D and Jemal A: Cancer statistics, 2013. CA Cancer J Clin 63: 11-30, 2013.

2. Li D, Wu C, Zheng Y, Zhong W and Wu F: Incidence and mortality of colorectal cancer in Shanghai from 2003 to 2007. Zhongguo Zhongliu 20: 413-418, 2011.

3. Woolf CM: A genetic study of carcinoma of the large intestine. Am J Hum Genet 10:42-47, 1958.

4. Winawer SJ, Zauber AG, Gerdes H, O'Brien MJ, Gottlieb LS, Sternberg SS, Bond JH, Waye JD, Schapiro M and Panish JF: Risk of colorectal cancer in the families of patients with adenomatous polyps National Polyp Study Workgroup. N Engl J Med 334: 82-87, 1996.

5. Magrone T and Jirillo E: The interaction between gut microbiota and age-related changes in immune function and inflammation. Immun Ageing 10: 31, 2013.

6. O'Toole PW: Changes in the intestinal microbiota from adulthood through to old age. Clin Microbiol Infect 18: 44-46, 2012.

7. DiBaise JK, Frank DN and Mathur R: Impact of the gut microbiota on the development of obesity: Current concepts. Am J Gastroenterol 1 (Suppl): 22-27, 2012.

8. Kallus SJ and Brandt LJ: The intestinal microbiota and obesity. J Clin Gastroenterol 46: 16-24, 2012.

9. Rubinstein MR, Wang X, Liu W, Hao Y, Cai GF and Han YW: Fusobacterium nucleatum promotes colorectal carcinogenesis by modulating e-cadherin/ $\beta$-catenin signaling via its FadA adhesin. Cell Host Microbe 14: 195-206, 2013.

10. Sobhani I, Amiot A, Le Baleur Y, Levy M, Auriault M, Van Nhieu JT and Delchier JC: Microbial dysbiosis and colon carcinogenesis: could colon cancer be considered a bacteria-related disease?. Therap Adv Gastroenterol 6: 215-229, 2013.

11. Tjalsma H, Boleij A, Marchesi JR and Dutilh BE: A bacterial driver-passenger model for colorectal cancer: Beyond the usual suspects. Nat Rev Microbiol 10: 575-582, 2012.

12. Ley RE, Peterson DA and Gordon JI: Ecological and evolutionary forces shaping microbial diversity in the human intestine. Cell 124: 837-848, 2006.

13. Purchiaroni F, Tortora A, Gabrielli M, Bertucci F, Gigante G, Ianiro G, Ojetti V, Scarpellini E and Gasbarrini A: The role of intestinal microbiota and the immune system. Eur Rev Med Pharmacol Sci 17: 323-333, 2013.

14. Xu X, Xu P, Ma C, Tang J and Zhang X: Gut microbiota, host health and polysaccharides. Biotechnol Adv 31: 318-337, 2013.

15. Compare D and Nardone G: The bacteria-hypothesis of colorectal cancer: Pathogenetic and therapeutic implications. Transl Gastrointest Cancer 3: 44-53, 2014.

16. Rowland I: Probiotics and colorectal cancer risk. Br J Nutr 91: 805-807, 2004.

17. Uccello M, Malaguarnera G, Basile F, D'agata V, Malaguarnera M, Bertino G, Vacante M, Drago F and Biondi A: Potential role of probiotics on colorectal cancer prevention. BMC Surg 12: S35, 2012.

18. Delia P, Sansotta G, Donato V,Frosina P, Messina G, De Renzis C and Famularo G: Use of probiotics for prevention of radiation-induced diarrhea. World J Gastroenterol 13: 912-915, 2007. 
19. Famularo G, Mosca L, Minisola G, Trinchieri V and De Simone C: Probiotic lactobacilli: A new perspective for the treatment of inflammatory bowel disease. Curr Pharm Des 9: 1973-1980, 2003.

20. Ljungh A and Wadström T: Lactic acid bacteria as probiotics. Curr Issues Intest Microbiol 7: 73-89, 2006.

21. Allen SJ, Wareham K, Wang D, Bradley C, Hutchings H, Harris W, Dhar A, Brown H, Foden A, Gravenor MB, et al: Lactobacilli and bifidobacteria in the prevention of antibiotic-associated diarrhoea and Clostridium difficile diarrhoea in older inpatients (PLACIDE): A randomised, double-blind, placebo-controlled, multicentre trial. Lancet 382: 1249-1257, 2013.

22. Mackowiak PA: Recycling metchnikoff: Probiotics, the intestinal microbiome and the quest for long life. Front Public Health 1: 52, 2013.

23. Allen SJ, Martinez EG, Gregorio GV and Dans LF: Probiotics for treating acute infectious diarrhoea. Cochrane Database Syst Rev 10: CD003048, 2010.

24. Chen CC, Kong MS, Lai MW, Chao HC, Chang KW, Chen SY, Huang YC, Chiu CH, Li WC, Lin PY, et al: Probiotics have clinical, microbiologic and immunologic efficacy in acute infectious diarrhea. Pediatr Infect Dis J 29: 135-138, 2010.

25. Hempel S, Newberry SJ, Maher AR, Wang Z, Miles JN, Shanman R, Johnsen B and Shekelle PG: Probiotics for the prevention and treatment of antibiotic-associated diarrhea a systematic review and meta-analysis. JAMA 307: 1959-1969, 2012.

26. Koebnick C, Wagner I, Leitzmann P, Stern U and Zunft HJ: Probiotic beverage containing Lactobacillus casei Shirota improves gastrointestinal symptoms in patients with chronic constipation. Can J Gastroenterol 17: 655-659, 2003.

27. Ouwehand AC, Lagström H, Suomalainen T and Salminen S: Effect of probiotics on constipation, fecal azoreductase activity and fecal mucin content in the elderly. Ann Nutr Metab 46 : 159-162, 2002

28. de Milliano I, Tabbers MM, van der Post JA and Benninga MA Is a multispecies probiotic mixture effective in constipation during pregnancy? 'A pilot study'. Nutr J 11: 80, 2012.

29. Persborn M and Söderholm JD: Commentary: the effects of probiotics on barrier function and mucosal pouch microbiota during maintenance treatment for severe pouchitis in patients with ulcerative colitis-authors' reply. Aliment Pharmacol Ther 38: 1406-1407, 2013.

30. Tursi A, Brandimarte G, Papa A, Giglio A, Elisei W, Giorgetti GM, Forti G, Morini S, Hassan C, Pistoia MA, et al: Treatment of relapsing mild-to-moderate ulcerative colitis with the probiotic Vs.L\#3 as adjunctive to a standard pharmaceutical treatment: A double-blind, randomized, placebo-controlled study. Am J Gastroenterol 105: 2218-2227, 2010.

31. Müllner K, Miheller P, Herszényi L and Tulassay Z: Probiotics in the management of Crohn's disease and ulcerative colitis. Curr Pharm Des 20: 4556-4560, 2014.

32. Huebner C, Ding Y, Petermann I, Knapp C and Ferguson LR: The probiotic Escherichia coli Nissle 1917 reduces pathogen invasion and modulates cytokine expression in Caco-2 cells infected with Crohn's disease-associated E. coli LF82. Appl Environ Microbiol 77: 2541-2544, 2011

33. Kõljalg U, Nilsson RH, Abarenkov K, Tedersoo L, Taylor AF, Bahram M, Bates ST, Bruns TD, Bengtsson-Palme J, Callaghan TM, et al: Towards a unified paradigm for sequence-based identification of fungi. Mol Ecol 22: 5271-5277, 2013.
34. Hamady M, Walker JJ, Harris JK, Gold NJ and Knight R: Error-correcting barcoded primers for pyrosequencing hundreds of samples in multiplex. Nat Methods 5: 235-237, 2008.

35. Edgar RC: UPARSE: Highly accurate OTU sequences from microbial amplicon reads. Nat Methods 10: 996-998, 2013.

36. Quast C, Pruesse E, Yilmaz P, Gerken J, Schweer T, Yarza P, Peplies J and Glöckner FO: The SILVA ribosomal RNA gene database project: Improved data processing and web-based tools. Nucleic Acids Res 41: D590-D596, 2013.

37. DeSantis TZ, Hugenholtz P, Larsen N, Rojas M, Brodie EL, Keller K, Huber T, Dalevi D, Hu P and Andersen GL: Greengenes, a chimera-checked 16S rRNA gene database and workbench compatible with ARB. Appl Environ Microbiol 72: 5069-5072, 2006.

38. Appleyard CB, Cruz ML, Isidro AA, Arthur JC, Jobin C and De Simone C: Pretreatment with the probiotic VSL\#3 delays transition from inflammation to dysplasia in a rat model of colitis-associated cancer. Am J Physiol Gastrointest Liver Physiol 301: G1004-G1013, 2011

39. Fotiadis CI, Stoidis CN, Spyropoulos BG and Zografos ED: Role of probiotics, prebiotics and synbiotics in chemoprevention for colorectal cancer. World J Gastroenterol 14: 6453-6457, 2008.

40. Wollowski I, Rechkemmer G and Pool-Zobel BL: Protective role of probiotics and prebiotics in colon cancer. Am J Clin Nutr 73: 451S-455S, 2001.

41. Bertkova I, Hijova E, Chmelarova A, Mojzisova G, Petrasova D, Strojny L, Bomba A and Zitnan R: The effect of probiotic microorganisms and bioactive compounds on chemically induced carcinogenesis in rats. Neoplasma 57: 422-428, 2010.

42. Vipperla K and O'Keefe SJ: The microbiota and its metabolites in colonic mucosal health and cancer risk. Nutr Clin Pract 27: 624-635, 2012

43. Wang J, Tang H, Zhang C, Zhao Y, Derrien M, Rocher E, vanHylckama Vlieg JE, Strissel K, Zhao L, Obin M and Shen J: Modulation of gut microbiota during probiotic-mediated attenuation of metabolic syndrome in high fat diet-fed mice. ISME 9: 1-15, 2015.

44. Kostic AD, Gevers D, Pedamallu CS, Michaud M, Duke F, Earl AM, Ojesina AI, Jung J, Bass AJ, Tabernero J, et al: Genomic analysis identifies association of Fusobacterium with colorectal carcinoma. Genome Res 22: 292-298, 2012.

45. Wang T, Cai G, Qiu Y, Fei N, Zhang M, Pang X, Jia W, Cai S and Zhao L: Structural segregation of gut microbiota between colorectal cancer patients and healthy volunteers. ISME J 6: 320-329, 2012.

46. Allen-VercoeE,Strauss J and ChadeeK: Fusobacteriumnucleatum: An emerging gut pathogen? Gut Microbes 2: 294-298, 2011.

47. Strauss J, Kaplan GG, Beck PL, Rioux K, Panaccione R, Devinney R, Lynch T and Allen-Vercoe E: Invasive potential of gut mucosa-derived Fusobacterium nucleatum positively correlates with IBD status of the host. Inflamm Bowel Dis 17: 1971-1978, 2011.

48. Arthur JC and Jobin C: The struggle within: Microbial influences on colorectal cancer. Inflamm Bowel Dis 17: 396-409, 2011.

49. Chaikham P and Apichartsrangkoon A: Effects of encapsulated Lactobacillus acidophilus along with pasteurized longan juice on the colon microbiota residing in a dynamic simulator of the human intestinal microbial ecosystem. Appl Microbiol Biotechnol 98: 485-495, 2014. 